\title{
A deep sea mission to seek out new lab animals
}

An international group of scientists has returned from the depths of the ocean with a new lab animal in hand: a glowing anemone whose tentacles contain a novel fluorescent protein.

Green fluorescent protein (GFP), which was first isolated in the 1960 s from a crystal jelly (Aequorea victoria), has become instrumental to our understanding of biological phenomena. By engineering cells to express GFP-like proteins, scientists can use imaging technology to visually track molecular processes. Fluorescent proteins have received heightened attention in recent weeks; the 2008 Nobel Prize in Chemistry was awarded to three scientists who were separately involved in the discovery, application and enhancement of these molecular markers.

GFPs have been isolated from several marine invertebrates, and different proteins have different photogenic properties. Some proteins can be switched on and off, and others can be induced to fluoresce at different colors. Fluorescent proteins can be enhanced with protein engineering, but to develop proteins with entirely

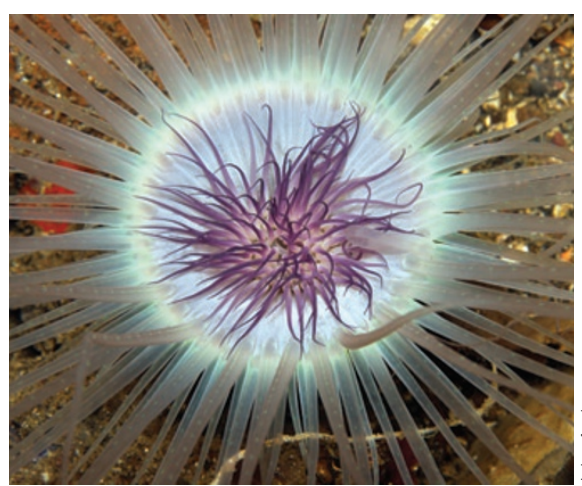

would trigger fluorescence in any GFPs encountered along the way (PLoS ONE 3 , e3766; 2008). The group discovered a luminescent ceriantharian (tube anemone), which may be a new species, though this remains to be confirmed. They isolated a novel GFP (called cerFP505) from the animal's tentacles. The new protein can be switched on and off by alternating irradiation with blue and with near-UV light, and its peak fluorescence is emitted at an irradiation of $505 \mathrm{~nm}$.

The investigators transfected a human cell line with cerFP505 and

new spectral properties, new natural protein sources must be discovered. Many GFP-expressing animals have been found in sunlit habitats such as tropical coral reefs. A group of scientists led by Joerg Wiedenmann (National Oceanography Institute, Southampton, UK), Mikhail Matz (University of Texas, Austin) and Charles Mazel (NightSea, Andover, MA) set out to search for light-emitting species in the dark depths of the sea.

The scientists explored the floor of the Gulf of Mexico in a submarine, which was equipped with blue lights that found the GFP to be effective as a cellular marker, with brightness and stability similar to those observed in commonly used fluorescent proteins. This is despite the fact that the protein was isolated from a species adapted to complete darkness and low temperatures, conditions that would be hostile to mammals. Thus, according to the scientists, the discovery "reveals the lightless depths of the oceans as a new reservoir of proteins with novel and highly desirable properties for imaging applications."

\section{Karen Marron}

\section{VACCINATING AGAINST AUTOIMMUNITY?}

Vaccines are already commonly used to prevent a variety of infectious diseases. Now they may become a key therapeutic strategy for a whole new host of disorders: autoimmune. New research from Peter Terness, Thilo Oelert, Sandra Ehser and their groups at Heidelberg University Hospital and German Cancer Research Center (Heidelberg, Germany) shows some success in preventing development of encephalitis in vaccinated mice, which is similar to the autoimmune disease multiple sclerosis (MS) in humans.

In autoimmune disease, the body's immune system responds to its own cells, producing antibodies to attack itself. This makes treatment of autoimmune diseases challenging. Existing treatments frequently suppress the immune response overall to halt disease progression, leaving the body susceptible to infection, or focus on the symptoms rather the underlying condition. A more ideal treatment for autoimmune disease would minimize the autoimmune reaction but preserve the rest of the immune response.

Terness and his team focus mainly on transplantation, researching ways to prevent rejection of transplanted organs or tissues without compromising the recipient's immune system. In previous work, they treated immune cells from a donor rat with mitomycin C (MMC), a chemotherapeutic drug; injected the treated cells into a recipient rat; and then transplanted heart tissue from the same donor to the recipient. In that study, the MMC-treated cells did not induce an immune reaction in the recipient, and the transplanted heart tissue was tolerated (Transplantation 83, 347-350; 2007).

According to Terness, the new vaccine they are developing against MS works in a similar manner to 'teach' the immune system not to fight against its own nerve cells. In this study, the scientists extracted immune cells from mice and loaded them with myelin basic protein, a component of the nervous system, which is the target of immune attack in MS. The cells were then treated with $M M C$ and injected back into the same mice. This process rendered the mice resistant to experimental autoimmune encephalitis (Proc. Natl. Acad. Sci. USA 105, 18442-18447; 2008), a disease that mirrors MS in humans and is frequently used as a model for MS research.

Having established that their strategy can prevent the development of encephalitis in mice, Terness's group plans to continue this line of research by investigating whether it is also effective in treating encephalitis in mice that have already developed the disease. Monica Harrington 